\title{
The Effect of Attack Angle On The Vibration Suppression Of Composite Wing Airfoil NACA 0012
}

\author{
Hassan Ali Kadhem ${ }^{1}$, Ahmed Abdul Hussein',* \\ 1'Baghdad University, baghdad, Iraq, Hali2013201359@gmail.com \\ ${ }^{2}$ Baghdad University, baghdad, Iraq, Dr.Ahmed.ali@coeg.uobaghdad.edu.iq \\ *corresponding author: Hassan Ali Kadhem Hali2013201359@gmail.com \\ Published online: 31 December 2020
}

Abstract- Active vibration control is presented as an effective technique used for vibration suppression and for attenuating bad effects of disturbances on structure. In this work Proportional-Integral-Derivative control were employed to study suppression of active vibration wing affected by wind airflow. Two different composite wings with different manufacturing materials had been made with specific size to be suitable for using in wind tunnel. Piezoelectric (PZT) transducers are used as sensors and actuators in vibration control systems. The velocity was $25 \mathrm{~m} / \mathrm{s}$ and three different attack angles $(0,10,20$ degrees) had been taken to show their effect on the wings vibrations suppression. The results shows that the suppression of the wing amplitude is reduced when the attack angle increases for both woven and random composite wing matt and this happened due to the vortex which became more violent at the increase of attack angle and also due to the area that face the wind which will increase when the attack angle increase and this will reduces the suppression. The maximum control amplitude of woven Glass-fiber matt was $1.75 \mathrm{~cm}$ and the damping was about $38 \%$ at zero attack angle while it was $2 \mathrm{~cm}$ and the damping was about $26 \%$ at 20 degree attack angle for random Glass-fiber composite matt.

Keywords - vibration suppression, Attack angle, Piezoelectric, NACA 0012, PID Control.

\section{Introduction}

Aeroelasticity is a science that deals with the interaction of aerodynamic forces and structural deformations. Simply aeroelasticity can be defined as the study of the interaction of inertial, structural and aerodynamic forces on aircraft, buildings, surface vehicles etc. When a structure moves through the air, the motion will cause aerodynamic loads, leading to deformations of the structure. The deformation inturn has an impact on the airflow, thus changing the aerodynamic loading. Apparently, there is a closed loop of aerodynamic and structural interactions, and depending on the properties of the structure and the airflow different methods of controlling had been used with many applications to give the reader enough knowledge about it. It is most important to presents previous works that remarked on active vibration control techniques. Zhi-Guang Song and Li F. (2012), Derivative of velocity and proportional feedback of two different types of controllers in vibration were used with PZT as sensors and actuator. They studied the ability of suppression of composite plate used for aero elastic analysis. No experimental tests had been presented and the modal analysis of tested model was formulated by MATLAB codes. Results show that proportional regulator has high effectiveness in vibration suppression of $31 \%$ and $25 \%$ for free and forced responses respectively [7]. Xingjian Dong, et al (2014), Composite plate with PZT was used to perform controlling loop. The study was aimed to evaluating the performance of an active regulator for suppression of undesired vibrations, this suppression was carried out via controlling loop and PZT as sensors and actuators totally formulated in FE environment. Numerical simulation had been done in ANSYS environment for composite smart plate equipped with PZT. Besides FE model, real model was fabricated and tested experimental for vibration suppression. About $59 \%$ of overall model vibration was suppressed with good agreement between both experimental and numerical responses [9] Riessom W., et al (2014), In the present study used PZT to sense the strain of flexible aluminum cantilever beam and fed it back to regulator 
loop for actively attenuation of its free oscillation. Finite element simulation was performed besides experiments to check the acceptance of measurements. Using NI (National Instruments) cRIO 9022 in LabView was used in controlling loop. Results exhibit high level of agreement between both tests. High performance of feedback regulator to attenuate about $49 \%$ of free oscillation was noticed. The control law has demonstrated $53.91 \%$ and $62.5 \%$ reduction in vibration for the first and second mods respectively [6]. M. Kerboua, et al (2015), the unforced oscillation of any structure is attenuated by its inherently damping properties. The work invoked to present a method called "passive piezoelectric vibration shunt control". In the beginning, the numerical solver was utilized in order to estimate best design and position of PZT. Maximum attenuation that was satisfied in oscillation was leveled by percent of forty two from bending oscillation. The results shown that the efficiency of the control is sensitive to the PZT patch location and the accuracy of the shunt circuit being tuned [1]. The main objective of our research is to show the effect of changing the angle of attack on the suppression of the two composite wing of airfoil NACA 0012 when the velocity of the wind was $25 \mathrm{~m} / \mathrm{s}$.

\section{Wings model}

Two composite wings were fabricated by skinning foam model. The foam model were made by cutting the foam via hot wire by passing the hot wire around the aluminum airfoil's shape getting from $\mathrm{CNC}$ machine as shown in Figure 1 .The two foam models were skinned by Glassfiber matt one with random and the other with woven ( $\{0 / 90\}$ Glass-fiber). Two layer of Glass-fiber matt for each random and woven .Every layer were coated by mixture of Resin, Thickener powder and Hardener. The two wings dimensions are as shown in Figure 2.

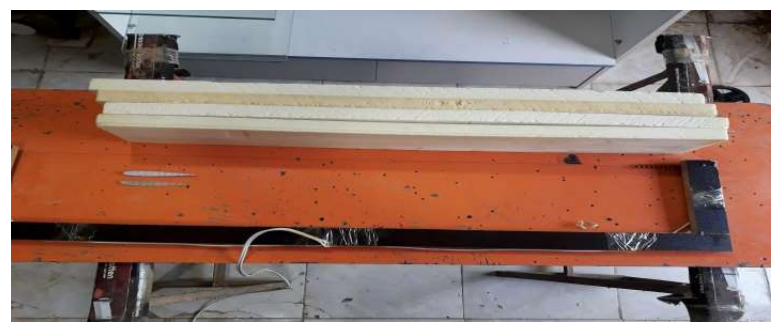

Figure 1: Show the hot wire and foam block
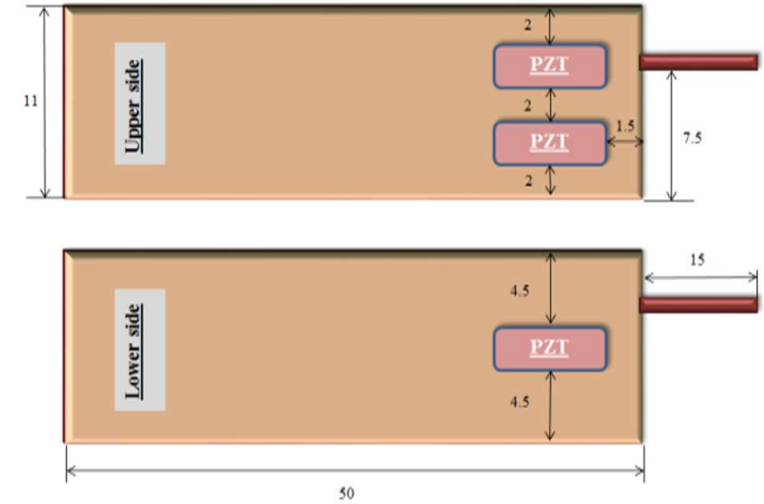

Figure 2: diagram show the diemnsion of the wing.

\section{Measuring Device}

\subsection{PiezoelectricTransducer}

Electrical transducer PPA-1001 can be used in this study as sensor and actuator. MID' piezo (standard products, USA) utilize its Piezo protection advantage (PPA) to protect the piezo ceramic wafers. The PPA- 1001 is a single layer product recommended for actuating, energy harvesting and sensing applications. It also exhibits good performance as a resonant actuator [5]. PPA-1001 is presented in Figure 3.

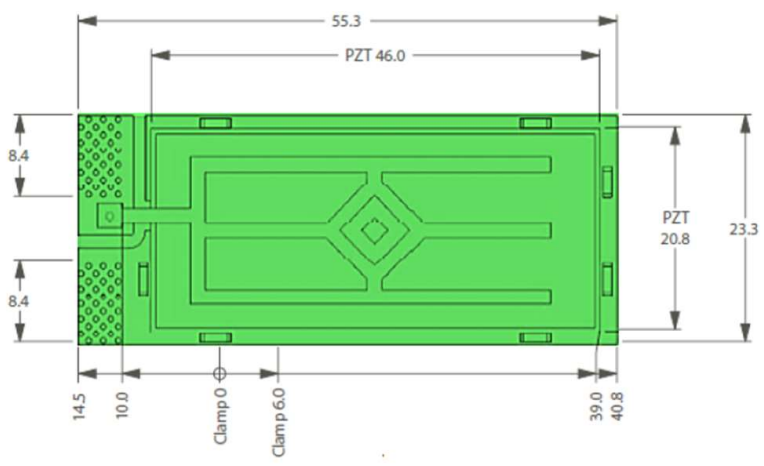

Figure 3: PPA-1001 transducer its dimension in (mm)

\subsection{Compact DAQ-9178 chassis (National Instruments)}

It is an 8-slot (National Instruments .USA) compact DAQ, USB chassis as shown in Figure 4 is designed for mixed-measurement, small, portable test systems. It combined sensor measurements with voltage, current, and digital signals to create custom mixed system with simple USB cable back to personal computer [2]. 


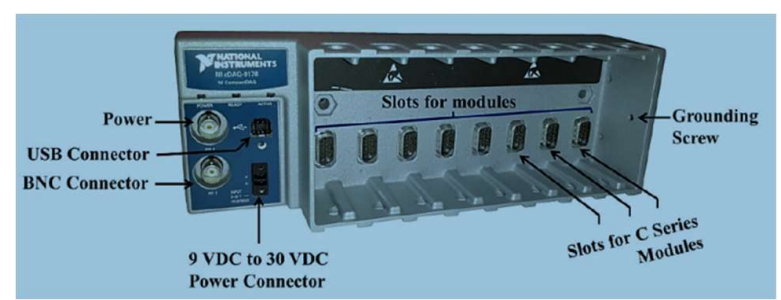

Figure 4: NI compact DAQ -9178.

\subsection{Analog input (NI 9215)}

Module compatible with NI compact-DAQ chassis contains four simultaneous sampled analog input channels. Channel to earth ground double isolation barrier with NIST-traceable calibration are included for system safety and noise filtering respectively. Also high range of voltage is available common mode. Range of Analog input is $\pm 10 \mathrm{~V}, 100 \mathrm{Ks} / \mathrm{s}$ per channel with 16-bit sampling rate resolution [3]. NI 2915 was designed to work within temperature range of $(-40 \sim 70)$. NI 9215 is one of national instrument products and it is presented in Figure 5.

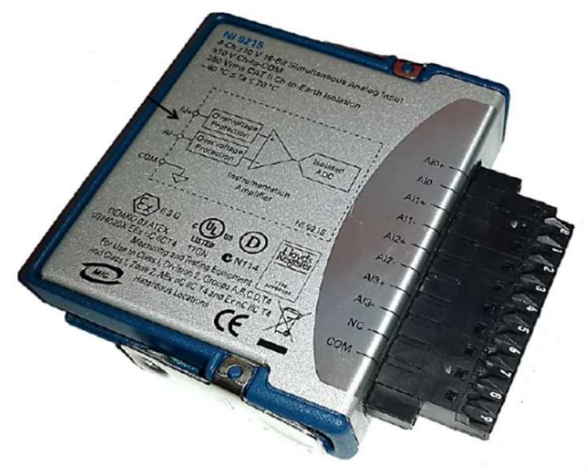

Figure 5: NI 9215- Analog input voltage.

\subsection{Signal Amplifier}

Two signal amplifiers were used for actuators. The devices used in this work are high voltage inverted operational amplifier (model 2205.USA) presented in Figure 6. Each amplifier had the ability to amplify voltage up to $\pm 500 \mathrm{~V}$, output current range and bandwidth are $\pm 80 \mathrm{~mA}$ and $75 \mathrm{kHz}$ with $3 \mathrm{~dB}$ respectively with maximum output power of $40 \mathrm{~W}$. Safety of measurements against overloading the actuator was provided by such amplifier to protect both amplifier circuit \& PZT from any damage caused by high input voltage or power [8].

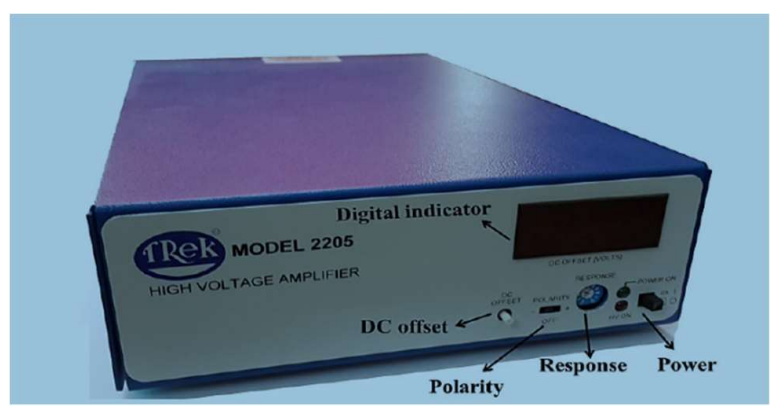

Figure 6: Signal amplifier.

\section{LabVIEW Program}

The active control program is developed in LabVIEW and it is used to apply active vibration. LabVIEW contains an inclusive set of tools for storing data, acquiring, and analyzing. In LabVIEW software the signal input is received and processing then sent as output signal at step time $(0.001 \mathrm{~s})$ to avoid shifting between the signals input and output [4]. Program of controller used in this work is presented in Figure 7.

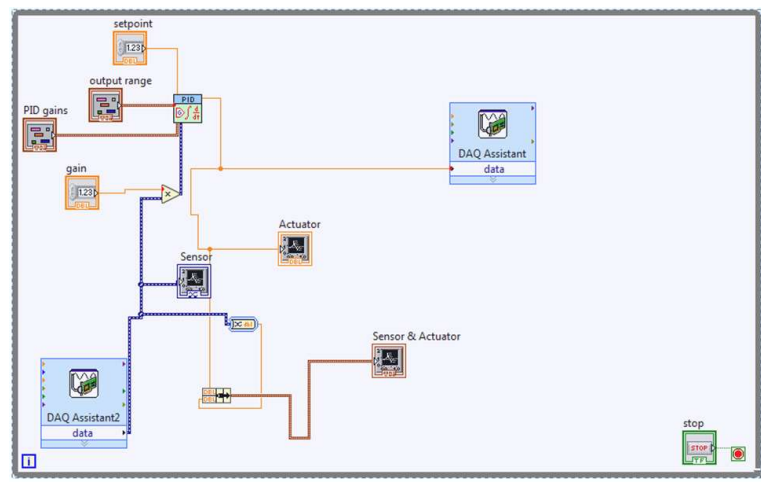

Figure 7: Block diagram of the controller used

\section{Experimental Work}

The experimental system used in this study shown clearly in Figure 8. The displacement responses of tested wing was sensed as voltage by piezoelectric, then sensed voltage (displacement) data is forwarded to analog input (NI 9215) which was used for noise filtering and acquiring date and by importing the relation between sensed voltage and displacement, voltage will be converted to its real value in lab view through DAQ which is used to convert sensed signal from analog to digital signal. The output actuators' signals are simultaneously sent through DAQ to analog output card. Then the controlling actuators' signals are sent to high voltage amplifier to drive the piezoelectric actuators which will act by opposite action to wing vibration to damping the vibration of the wing. 


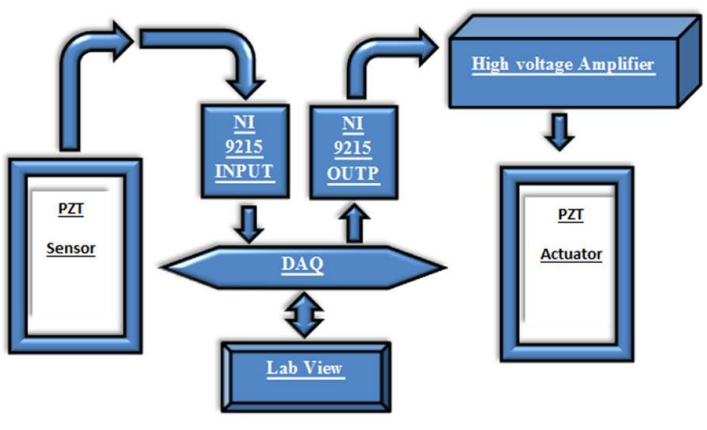

Figure 8: the diagram of experimental active vibration control.

\section{Results and discussions}

It had been notice that the suppression of the wing amplitude reduced when the attack angle increase for both woven and random composite wing matt as comparing with the free response and this happened due to the vortex which became more violent at the increase of attack angle and also due to the area that face the wind will increase when the attack angle increase and this will reduces the suppression as can see in figure 9and 10.

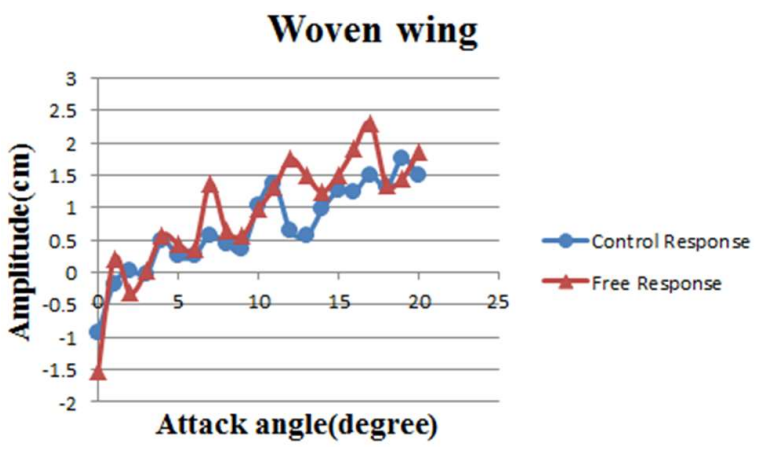

Figure 9: Relation of attack angle and Controlling Aplitude

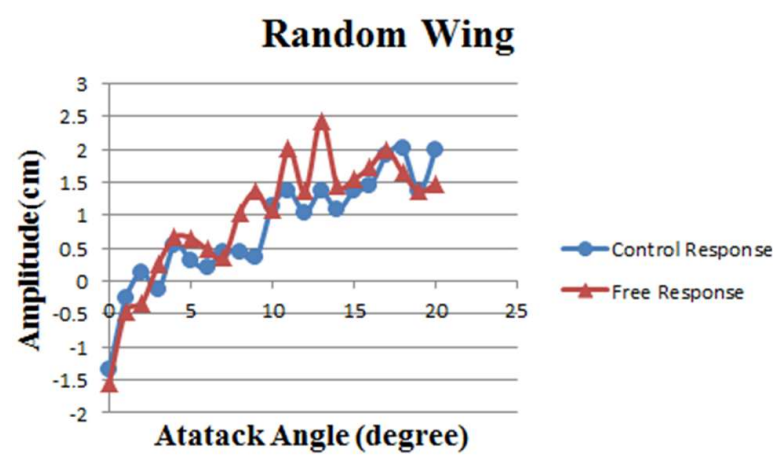

Figure10: Relation of attack angle and Controlling Amplitude

\section{Conclusion}

The suppression of the wing amplitude reduce when the attack angle increase for both woven and random composite wing matt. The composite wing which is made of woven Glass-fiber matt has high resistance more than random Glass-fiber composite matt and maximum control amplitude of woven Glass-fiber matt was $1.75 \mathrm{~cm}$ and the damping was about $38 \%$ at zero attack angle while it was $2 \mathrm{~cm}$ and the damping was about $26 \%$ at 20 degree attack angle for random Glass-fiber composite matt.

\section{References}

[1] Kerboua M, Megnounif A, Benguediab $M$, Benrahou KH, Kaoulala MF. "Vibration Control beam using Piezoelectric-based Smart Materials", Composite Structures, vol. 123, 2015, pp. 430-442.

[2] National Instrument, "Data sheet of Compact DAQ-9178 chassis", USA, 2014.

[3] National Instrument, "Data sheet of DAQ-NI 9215”, USA, 2014.

[4] National Instrument, "Lab VIEW-Control Design User Manual”, 2010.

[5] PPA-1001, Data Sheet, MIDE Co. Ltd., PPA-series 2016.

[6] Riessom W, Rao P, Scholar PG, Krishina P, Gangadharan K V. "Strain Feedback Active Vibration Control of Smart Cantilever Beam", vol. 5, 2014, pp. 113-122.

[7] Song Z, Li F. “Active Aeroelastic Flutter Analysis and Vibration Control of Supersonic Composite Laminated Plate", Composite Structures, vol. 94, 2012, pp. 702-13.

[8] Trek Inc., “Operator's Manual, Model 2205”, Piezo Driver/Power Amplifier", USA, 2013.

[9] Xingjian Dong, Zhike Peng, Lin Ye, Hongxing Hua and Guang Meng., "Performance Evaluation of Vibration Controller for Piezoelectric Smart Structures in Finite Element Environment", Journal of Vibration and Control,vol.20,2014. 


\title{
تأثيرزاوية الهجوم على تخميد اهتزازات جناح مركب ذو برفايل نوع NACA 0012
}

\author{
حسن علي كاظمب، /حد عب الحسبن 2، \\ Hali2013201359@gmail.com ، جامعة بغداد،بغد/د،العراق \\ Dr.Ahmed.ali@coeg.uobaghdad.edu.iq جامعة بغداد،بذ/د،/العراق، \\ "الباحث المثل: حسن علي كاظم،
}

$$
\text { نشر في: } 31 \text { كانون الاول } 2020
$$

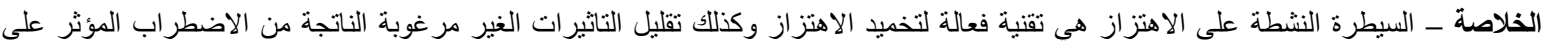

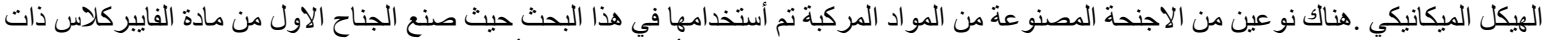

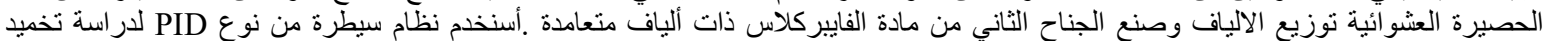

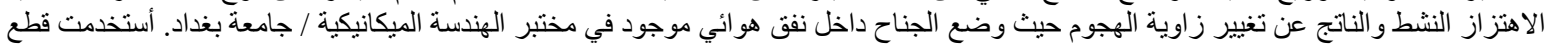

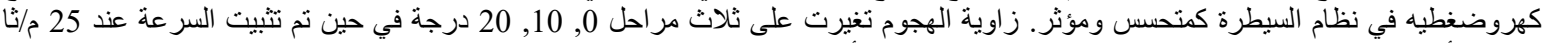

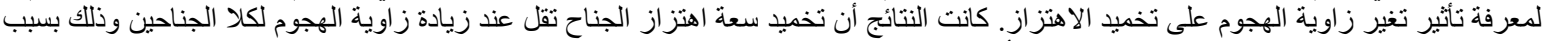

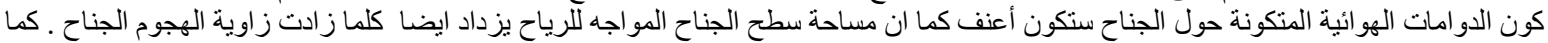

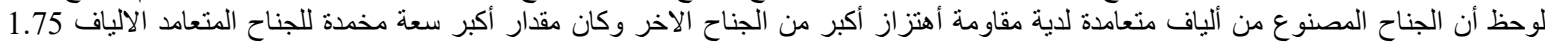

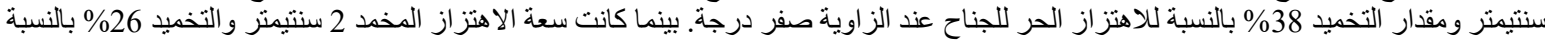

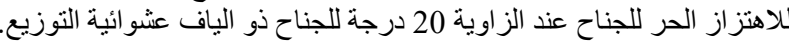

الكلمات الرئيسية -" زاوية الهجوم ، سيطرة من نوع PID، حساسات كهرضغطيه ،بروفايل نوع NACA0012، 